\title{
Clinicopathological characteristics of peripheral clinical stage IA lung adenocarcinoma with high Ki-67 expression
}

\author{
Zhan Liu ${ }^{1}$, Hongxiang Feng ${ }^{1}$, Shanwu Ma ${ }^{1}$, Weipeng Shao ${ }^{1}$, Jun Zhang ${ }^{1}$, Zhaohua Zhang ${ }^{1}$, \\ Hongliang Sun ${ }^{2}$, Xinlei Gu ${ }^{3}$, Zhenrong Zhang ${ }^{1}$, Deruo Liu ${ }^{1}$ \\ ${ }^{1}$ Department of Thoracic Surgery, China-Japan Friendship Hospital, Peking University China-Japan Friendship School of Clinical Medicine, \\ Beijing, China; ${ }^{2}$ Department of Radiology, China-Japan Friendship Hospital, Peking University China-Japan Friendship School of Clinical Medicine, \\ Beijing, China; ${ }^{3}$ Department of Thoracic Surgery, Peking University International Hospital, Beijing, China \\ Contributions: (I) Conception and design: D Liu, Z Zhang; (II) Administrative support: D Liu, Z Zhang, H Feng; (III) Provision of study materials or \\ patients: Z Zhang, H Feng, H Sun; (IV) Collection and assembly of data: Z Liu, S Ma, W Shao, J Zhang, Z Zhang, H Sun, X Gu; (V) Data analysis \\ and interpretation: Z Liu, S Ma, W Shao, J Zhang, Z Zhang, X Gu; (VI) Manuscript writing: All authors; (VII) Final approval of manuscript: All \\ authors. \\ Correspondence to: Deruo Liu; Zhenrong Zhang. Department of Thoracic Surgery, Center for Respiratory Diseases, China-Japan Friendship Hospital, \\ No. 2 Yinghua East Road, Chaoyang District, Beijing 100029, China. Email: deruoliu@163.com; doctor_zzr@126.com.
}

Background: High Ki-67 expression is associated with poor prognosis in early-stage lung adenocarcinoma (LUAD). However, there are few studies on the associations between clinicopathological features and Ki67 proliferation index (PI). The study aimed to explore the clinicopathological characteristics of peripheral clinical stage IA LUAD with high Ki-67 expression.

Methods: A case-control study was carried out in China-Japan Friendship Hospital from January 2017 to December 2018. The clinicopathological features of patients were reviewed. Univariate and multivariate analyses were used to analyze the association between clinicopathological characteristics and high Ki-67 expression.

Results: Three hundred and seventy-six patients were finally enrolled in the study. Univariate and multivariate analyses showed that males sex $(\mathrm{OR}=2.23,95 \% \mathrm{CI}: 1.30-3.83, \mathrm{P}=0.004)$, carcinoembryonic antigen (CEA) positivity $(\mathrm{OR}=3.25,95 \% \mathrm{CI}: 1.44-7.33, \mathrm{P}=0.005)$, several imaging features such as notch positivity $(\mathrm{OR}=2.55,95 \% \mathrm{CI}: 1.18-5.51, \mathrm{P}=0.017)$, vascular convergence $(\mathrm{OR}=3.04,95 \% \mathrm{CI}: 1.03-8.95$, $\mathrm{P}=0.044)$, and consolidation/tumor ratio (CTR) $(\mathrm{OR}=1.03,95 \% \mathrm{CI}: 1.02-1.04, \mathrm{P}<0.001)$ were significantly associated with high Ki-67 expression. The area under curve of receiver operating characteristic (ROC) curve for CTR was 0.813 (95\% CI: $0.768-0.858, \mathrm{P}<0.001$ ). When cutoff value was $72.5 \%$, the sensitivity and specificity were $80.5 \%$ and $76.3 \%$, respectively.

Conclusions: Male sex, CEA positivity, notch positivity, vascular convergence, and CTR were significantly associated with high Ki-67 expression in patients with peripheral clinical stage IA LUAD. These findings could be used to assist clinical decision-making and prognostic evaluation.

Keywords: Lung adenocarcinoma (LUAD); Ki-67; computed tomography (CT); pathology

Submitted Jul 27, 2020. Accepted for publication Nov 20, 2020.

doi: $10.21037 /$ tcr-20-2608

View this article at: http://dx.doi.org/10.21037/tcr-20-2608 


\section{Introduction}

$\mathrm{Ki}-67$ is a kind of DNA-binding nuclear protein expressed throughout the proliferative phases of the cell cycle, but not in the quiescent phase (1). Therefore, it has become a wellknown biomarker to evaluate the proportion of proliferating cells in a specific cell population (2). In clinical studies, it has been confirmed that high $\mathrm{Ki}-67$ expression is closely related to a poor prognosis in a variety of tumors, such as breast cancer, gastrointestinal stromal tumor, pancreatic cancer, prostate cancer, and bladder cancer (3-7).

Lung cancer is the most common malignant tumor worldwide and one of the main causes of cancer-related death at present (8). In recent years, lung adenocarcinoma (LUAD) has been the most common pathological type of lung cancer, and its incidence has grown rapidly (9). High expression of $\mathrm{Ki}-67$ is significantly associated with a poor prognosis in patients with LUAD, including even earlystage LUAD (10-13). In addition, Ki-67 proliferation index (PI) is an independent risk factor for recurrence in patients with early-stage LUAD after segmentectomy (14). However, Ki-67 PI has not yet become a routine parameter evaluated by pathological detection in lung cancer patients. At present, there are few studies on the associations between clinicopathological features and Ki-67 PI.

Therefore, the study aimed to explore the clinicopathological characteristics of peripheral clinical stage IA LUAD with high $\mathrm{Ki}-67$ expression. We present the following article in accordance with the STROBE reporting checklist (available at http://dx.doi.org/10.21037/tcr-202608).

\section{Methods}

\section{Study population}

A retrospective case-control study was carried out in China-Japan Friendship Hospital from January 2017 to December 2018. The study was conducted in accordance with the Declaration of Helsinki (as revised in 2013). The study was approved by the institutional ethics committee of China-Japan Friendship Hospital (No. 2018-13-K08). The institutional ethics committee waived the need for informed consent because of its retrospective design and the use of anonymized data.

Clinical, pathological, and radiological features of patients who underwent curative operations for LUAD with clinical tumor size $\leq 3 \mathrm{~cm}$ were reviewed. Inclusion criteria: (I) according to the tumor-node-metastasis (TNM) stage in the 8th edition of lung cancer (15), the preoperative stage was cIA; (II) operations were performed, including lobectomy, segmentectomy and wedge resection of the lung, with mediastinal lymph nodes sampling or dissection; (III) LUAD was confirmed by pathological examination which was performed within 1 week after the operation. Exclusion criteria: (I) history of previous lung operation; (II) multiple pulmonary nodules on chest computed tomography (CT); (III) without mediastinal lymph node sampling or dissection; (IV) metastatic LUAD; (V) without definite pathological subtype or specific variants of invasive LUAD such as fetal or enteric LUAD; (VI) Ki-67 immunohistochemical staining was not performed after the operation.

\section{Clinical features}

The electronic medical records of all patients were reviewed, and the relevant clinical features were collected such as sex, age, smoking status, carcinoembryonic antigen (CEA) level, and operation procedures. The normal upper limit of CEA was $5 \mathrm{ng} / \mathrm{mL}$.

\section{Pathological features}

All patients' postoperative pathology reports were reviewed. The pathological features included pathological type, tumor tissue size, lymph node metastasis, pathological TNM stage, visceral pleura invasion (VPI), lymphovascular invasion (LVI), epidermal growth factor receptor (EGFR) gene mutation status, spread through air spaces (STAS), and Ki67 PI.

According to the classification method of the International Lung Cancer Research Association/American Thoracic Society/European Respiratory Society, invasive adenocarcinoma (IAD) was further classified (16). The pathological TNM stage was based on the eighth edition of TNM classification proposed by the International Association for the Study of Lung Cancer (IASLC) Lung Cancer Staging Project (15). According to the 2015 World Health Organization (WHO) classification of lung cancer, STAS was defined as pathological micropapillary clusters, solid nests or single cells beyond the edge of the tumor, and are separated from the main tumor (17).

The mouse anti-human Ki-67 monoclonal antibody (Zhongbin Jinqiao Biotechnology Co., Ltd., Beijing, China) was used for immunohistochemical staining in strict accordance with the instructions. Five high-power fields were randomly selected from each staining section. The 
average percentage of positive cells in the total number of cells was quantitatively analyzed as the Ki-67 PI. PI $\geq 14 \%$ was defined as high $\mathrm{Ki}-67$ expression and $\mathrm{PI}<14 \%$ was defined as low $\mathrm{Ki}-67$ expression.

\section{Radiological features}

All enrolled patients underwent high-resolution CT (HRCT) within 2 weeks preoperatively. HRCT was performed with the patient in the supine position during inspiratory breath-hold using various multidetector row scanners: Aqui-lion 4 (TOSHIBA Corporation, Tokyo, Japan), SOMA-TOM Plus4 Volume Zoom (SIEMENS, Munich, Germany), Brilliance CT (Philips, Amsterdam, the Netherlands). The imaging parameters were as follows: tube voltage, $120 \mathrm{kVp}$; tube current, $100-$ $150 \mathrm{~mA}$; detector collimation, $0.625-1.5 \mathrm{~mm}$; beam pitch, 1.375-1.5. Two experienced radiologists re-evaluated the chest CT images independently, without informing the relevant clinicopathological features, and reached a consensus through discussion in case of disagreement. The measurement data were all measured on the crosssectional images of the chest CT in picture archiving and communication systems (PACS) (lung window setting width, 1,500 HU and level, -600 HU) for three times, and the average value was taken.

Evaluation contents included the maximum nodule diameter, the maximum consolidation diameter, consolidation/tumor ratio (CTR), and the common CT features of peripheral lung cancer, such as cavitation, notch, spiculation, pleural indentation, air bronchogram, and vascular convergence. Ground-glass opacity (GGO) referred to the increased density of lung tissues without covering the original vascular and bronchial shadow that foils the region (18). The CTR was defined as (the maximum consolidation diameter/the maximum tumor diameter) $\times 100 \%$.

\section{Statistical analysis}

Statistical analyses were performed using IBM SPSS Statistics 22.0 (IBM, Chicago, IL, USA). Continuous variables with a normal distribution are presented as the mean \pm standard deviation (SD), nonnormally distributed variables are reported as the median (interquartile range). Categorical variables were represented by the number and percentage of cases. Clinicopathological feature differences between groups were compared by using the Student's $t$-test or Mann-Whitney $\mathrm{U}$ test for continuous variables, and $\chi^{2}$ test, continuous calibration $\chi^{2}$ test, or Fisher exact test for categorical variables. The variables with $\mathrm{P}<0.05$ in the univariate analysis were carried into binary Logistic regression analysis (forward stepwise regression manner). A receiver operating characteristic (ROC) curve was used to determine the optimal cutoff values for continuous variables. The area under the curve (AUC) was adopted to measure the diagnostic power. The Youden index was used to select the optimal sensitivity and specificity from the ROC curves. $\mathrm{P}<0.05$ was considered to indicate statistical significance (double-tailed).

\section{Results}

\section{Patient characteristics}

From January 2017 to December 2018, 807 patients who underwent curative operations for peripheral clinical stage IA LUAD were enrolled. Four hundred and thirtyone patients were excluded from the study cohort because of a history of previous lung operation $(n=12)$, multiple pulmonary nodules $(n=40)$, without mediastinal lymph node sampling or dissection $(n=98)$, metastatic LUAD $(n=7)$, specific variants of adenocarcinoma such as fetal or enteric adenocarcinoma $(n=4)$, without definite pathological subtype $(n=32)$, or without Ki-67 PI $(n=238)$. After exclusion, 376 patients (123 high Ki-67 expression patients and 253 low Ki-67 expression patients) were finally enrolled in this study (Figure 1).

The patients' baseline characteristics are summarized in Table 1. There were 137 males (36.4\%) and 239 females $(63.6 \%)$ with an average age of $(58 \pm 10)$ years old in the present study. Seventy-seven patients $(20.5 \%)$ had a smoking history, and 45 patients $(12.0 \%)$ were CEA positive. Three hundred and twenty-eight patients $(87.2 \%)$ underwent lobectomy, 23 patients $(6.2 \%)$ underwent segmentectomy, and 25 patients $(6.6 \%)$ underwent wedge resection.

Postoperative pathological reports showed that $\mathrm{Ki}$ 67 expression was high in 123 patients $(32.7 \%)$ and low in 253 patients $(67.3 \%)$. There were 1 patient with $(0.2 \%)$ adenocarcinoma in situ (AIS), 45 patients $(12.0 \%)$ with microinvasive adenocarcinoma (MIA), and 330 patients $(87.8 \%)$ with IAD. For invasive LUAD, the most prevalent subtype was acinar predominant $(58,15.4 \%)$, followed by lepidic predominant $(189,50.3 \%)$, papillary predominant $(41,10.9 \%)$, solid predominant $(25,6.6 \%)$, 


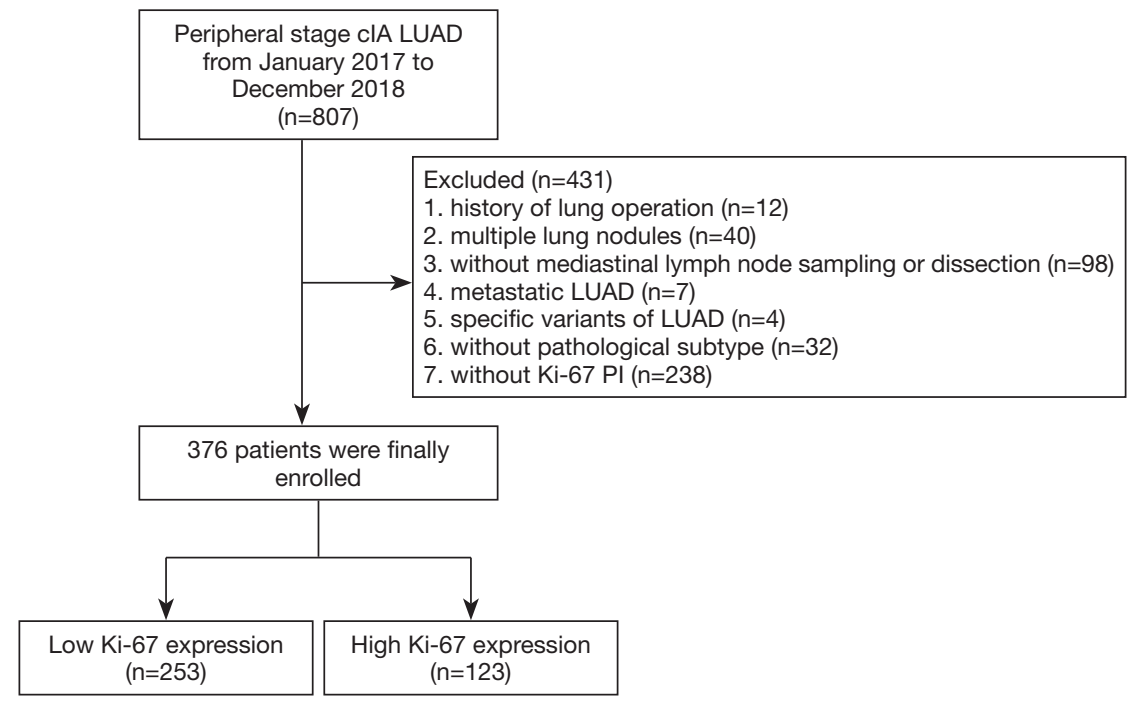

Figure 1 Flow chart for the study population. LUAD, lung adenocarcinoma.

Table 1 Association between Ki-67 PI and clinical features

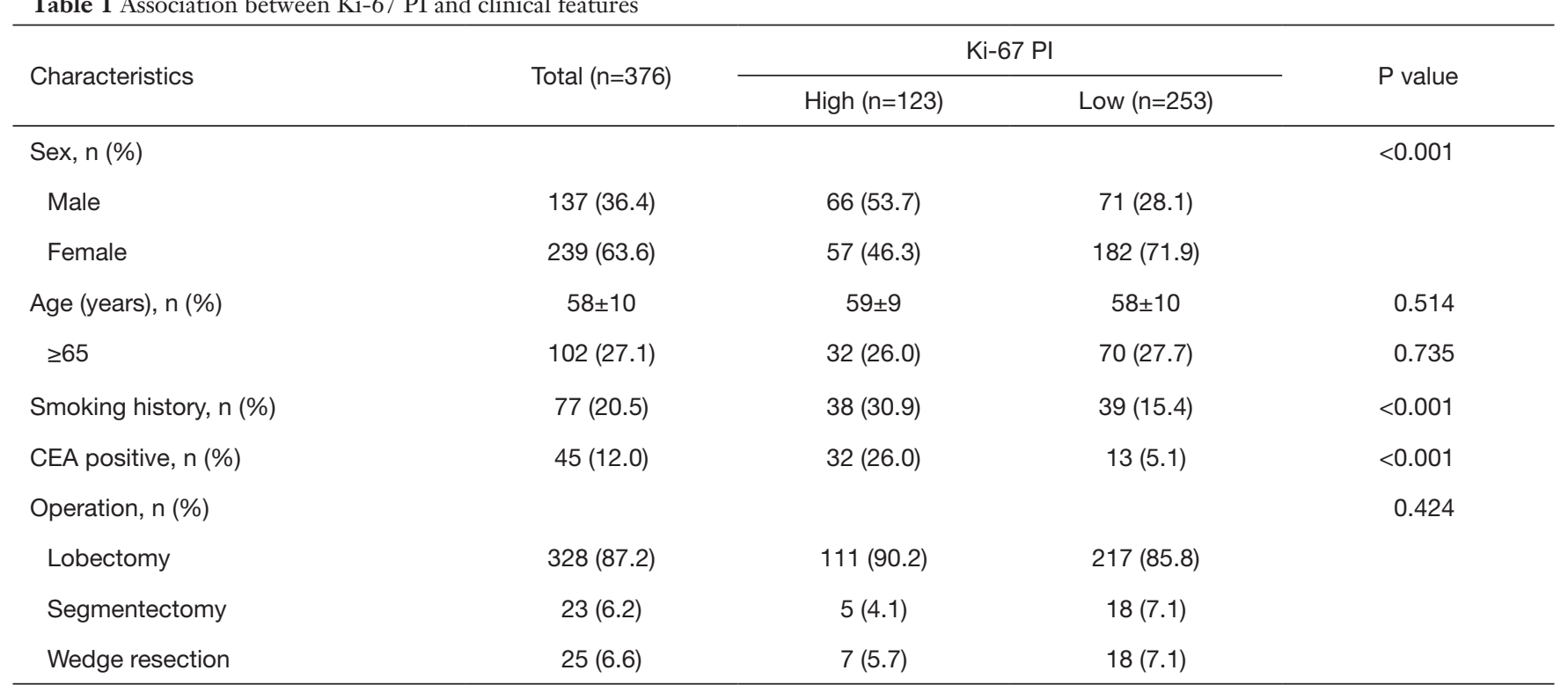

$\mathrm{PI}$, proliferation index; CEA, carcinoembryonic antigen.

mucinous adenocarcinoma (10, 2.7\%), and micropapillary predominant $(7,1.9 \%)$. There were 90 patients $(23.9 \%)$ with VPI positive, 15 patients (4.0\%) with LVI positive, and 54 patients $(14.4 \%)$ with STAS positive in the cohort. N1 and N2 lymph node involvement occurred in 17 (4.5\%) and 33 cases (8.8\%), respectively. Pathological stage I, II, and III tumors were found in 325 (86.4\%), 18 (4.8\%), and 33 $(8.8 \%)$ patients, separately. Three hundred and ten patients received EGFR gene molecular test, among which 174 patients (56.1\%) harbored EGFR gene mutations (Table 2).

In terms of radiological features, the maximum diameter of nodules was $16[8,16] \mathrm{mm}$, the maximum consolidation diameter was $8[0,17] \mathrm{mm}$, and the CTR was $54 \%[0$, 97\%]. Among the common CT features, cavitation, notch, spiculation, pleural indentation, air bronchogram, and vascular convergence were found in 39 (10.4\%), 27 (73.9\%), $241(64.1 \%), 183$ (65.0\%), 47 (12.5\%), and 335 (89.1\%) cases, respectively (Table 3). 
Table 2 Association between Ki-67 PI and pathological features



PI, proliferation index; AIS, adenocarcinoma in situ; MIA, microinvasive adenocarcinoma; IAD, invasive adenocarcinoma; VPI, visceral pleura invasion; LVI, lymphovascular invasion; STAS, spread through air spaces; EGFR, epidermal growth factor receptor; TNM, tumornode-metastasis.

\section{Univariate analysis for the association between clinicopathological characteristics and high Ki-67 expression}

The results of univariate analysis showed that patients with high Ki-67 expression were mostly males $(\mathrm{P}<0.001)$, who had a smoking history $(\mathrm{P}<0.001)$, and a higher CEA level $(\mathrm{P}<0.001)$ (Table 1). In terms of pathological features, high Ki-67expression was correlated with invasive LUAD $(\mathrm{P}<0.001)$, especially micropapillary predominant $(\mathrm{P}=0.040)$ and solid predominant (P 0.001) LUAD. However, low expression of $\mathrm{Ki}-67$ was more common in lepidic predominant $(\mathrm{P}<0.001)$ LUAD. In addition, high Ki67 expression was significantly associated with a larger pathological $\mathrm{T}$ stage $(\mathrm{P}<0.001)$, VPI positivity $(\mathrm{P}<0.001)$, LVI positivity $(\mathrm{P}<0.001)$, STAS positivity $(\mathrm{P}=0.003)$, lymph node metastasis $(\mathrm{P}<0.001)$ and a higher pathological TNM stage $(\mathrm{P}<0.001)$ (Table 2). In patients with high Ki67 expression, the maximum nodule diameter $(\mathrm{P}<0.001)$, 
Table 3 Association between Ki-67 PI and radiological features

\begin{tabular}{|c|c|c|c|c|}
\hline Characteristics & Total $(n=376)$ & \multicolumn{2}{|c|}{ Ki-67 PI } & $P$ value \\
\hline \multicolumn{5}{|l|}{ CT feature, $\mathrm{n}(\%)$} \\
\hline Cavitation & $39(10.4)$ & $16(13.0)$ & $23(9.1)$ & 0.242 \\
\hline Notch & 278 (73.9) & $109(88.6)$ & $169(66.8)$ & $<0.001$ \\
\hline Pleural indentation & $183(65.0)$ & $80(65.0)$ & $103(40.7)$ & $<0.001$ \\
\hline Air bronchogram & $47(12.5)$ & $21(17.1)$ & $26(10.3)$ & 0.062 \\
\hline Vascular convergence & $335(89.1)$ & $117(95.1)$ & $218(86.2)$ & 0.009 \\
\hline Maximum tumor diameter $(\mathrm{mm})^{\star}$ & $16[8,16]$ & $19[15,24]$ & $14[10,20]$ & $<0.001$ \\
\hline
\end{tabular}

*, non-normal continuous variables, the data were reported as median [interquartile range]. PI, proliferation index; CT, computed tomography; CTR, consolidation/tumor ratio.

Table 4 Multivariate analysis for the association between Ki-67 PI and clinicopathological features

\begin{tabular}{lcc}
\hline Characteristics & OR $(95 \% \mathrm{Cl})$ & P value \\
\hline Sex & 1.00 & 0.004 \\
Female & $2.23(1.30-3.83)$ & \\
$\quad$ Male & $3.25(1.44-7.33)$ & 0.005 \\
CEA positive & $2.55(1.18-5.51)$ & 0.017 \\
Notch & $3.04(1.03-8.95)$ & 0.044 \\
Vascular convergence & $1.03(1.02-1.04)$ & $<0.001$ \\
CTR &
\end{tabular}

PI, proliferation index; CEA, carcinoembryonic antigen; CTR, consolidation/tumor ratio.

consolidation diameter $(\mathrm{P}<0.001)$, and $\mathrm{CTR}(\mathrm{P}<0.001)$ were larger than patients with low Ki-67 expression, and several imaging features such as notch $(\mathrm{P}<0.001)$, spiculation $(\mathrm{P}<0.001)$, pleural indentation $(\mathrm{P}<0.001)$, and vascular convergence $(\mathrm{P}=0.009)$ were more common on chest $\mathrm{CT}$ imagines (Table 3).

\section{Multivariate analysis for the association between clinicopathological characteristics and high Ki-67 expression}

Variables with significant differences in univariate analysis were included in multivariate analysis. The results showed that males sex $(\mathrm{OR}=2.23,95 \% \mathrm{CI}: 1.30-3.83, \mathrm{P}=0.004)$, CEA positivity ( $\mathrm{OR}=3.25,95 \% \mathrm{CI}: 1.44-7.33, \mathrm{P}=0.005)$, and several imaging features such as notch positivity (OR $=2.55,95 \%$ CI: $1.18-5.51, \mathrm{P}=0.017)$, vascular convergence $(\mathrm{OR}=3.04,95 \%$ CI: $1.03-8.95, \mathrm{P}=0.044)$, and CTR (OR $=1.03,95 \%$ CI: $1.02-1.04, \mathrm{P}<0.001)$ were significantly associated with high Ki-67 expression (Table 4). The ROC curve showed that the AUC for CTR was 0.813 (95\% CI: $0.768-0.858, \mathrm{P}<0.001)$. When cutoff value was $72.5 \%$, the sensitivity and specificity were $80.5 \%$ and $76.3 \%$, respectively (Figure 2).

\section{Discussion}

Several clinical studies have confirmed that high Ki-67 expression is significantly related to a poor prognosis in a variety of tumors, such as breast cancer, gastrointestinal stromal tumor, pancreatic tumor, prostate cancer, and bladder cancer (3-7). Moreover, Ki-67 is also an important reference in clinical decision-making for patients with breast cancer or gastrointestinal neuroendocrine tumors $(19,20)$. However, given the limit related to the heterogeneity of the Ki-67 PI cutoff value, $\mathrm{Ki}-67$ has not become a routine pathological detection parameter for lung cancer in clinical work.

A meta-analysis including 108 studies showed that the cutoff value of Ki-67 PI was between 3\% and $75 \%$ and that 




Figure 2 ROC curve of CTR. The ROC curve showed that the AUC for CTR was 0.813 (95\% CI: 0.768-0.858, P<0.001). When cutoff value was $72.5 \%$, the sensitivity and specificity were $80.5 \%$ and $76.3 \%$, respectively. ROC, receiver operating characteristic; CTR, consolidation/tumor ratio; AUC, area under the curve.

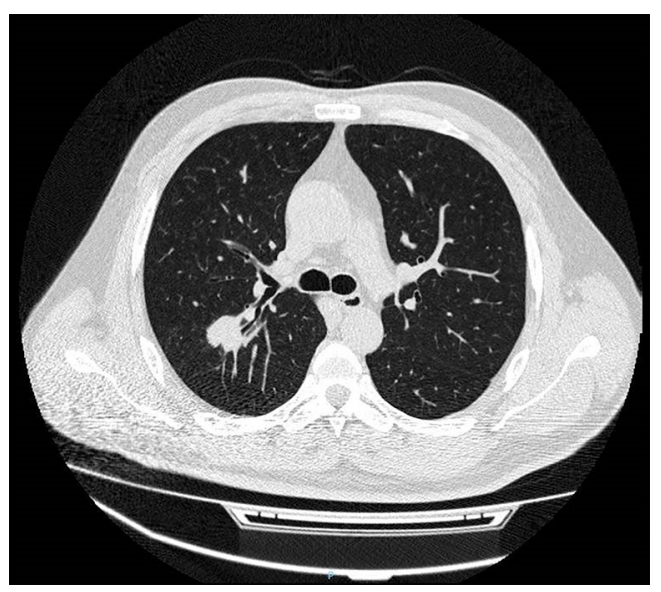

Figure 3 Representative CT images of the patient with high Ki67 expression. A 56-year-old male patient with pathologically confirmed LUAD with high Ki-67 expression. The Ki-67 PI was $60 \%$. Chest CT showed a pure solid nodule (CTR was 1.00) with notch, vascular convergence, and air bronchogram in the upper lobe of the right lung. CT, computed tomography; LUAD, lung adenocarcinoma; PI, proliferation index; CTR, consolidation/ tumor ratio.

the cutoff value of $\mathrm{Ki}-67 \mathrm{PI}$ was one of the main sources of heterogeneity (10). A study by Warth showed that in patients with NSCLC, high Ki-67 expression with a lower cutoff level indicated a poor prognosis, while opposing results were found with a higher cutoff level. Furthermore, high expression of $\mathrm{Ki}-67$ has different prognostic significance in different pathological types of NSCLC. In LUAD patients, high expression of Ki-67 indicates a poor prognosis, while, in contrast, it is related to a relatively good prognosis in lung squamous cell carcinoma patients (21). Therefore, independent evaluation of the expression of $\mathrm{Ki}-67$ in different pathological types of lung cancer and establishment of a unified Ki-67 PI cutoff value based on prognosis are urgently needed.

Our study found that the high expression of $\mathrm{Ki}-67$ was more common in males $(\mathrm{P}=0.004)$ and CEA-positive patients $(\mathrm{P}=0.005)$, which was consistent with previous reports $(10,22,23)$. Interestingly, some studies have shown that testosterone can promote the growth of cancer cells expressing androgen receptor (AR), while AR negatively regulates the Ki-67 level in lung cancer patients $(24,25)$. However, the current relevant mechanism is not clear at present, and further research is needed.

In the present study, high Ki-67 expression is significantly related to notch $(\mathrm{P}=0.017)$, vascular convergence $(\mathrm{P}=0.044)$, and CTR $(\mathrm{P}<0.001)$ (Figure 3). Yan et al. (26) described the associations between Ki-67 PI and radiological features in patients with LUAD and found that Ki-67 was correlated with notch and nodal density (Spearman correlation coefficient was 0.554 and 0.436 , respectively). This was consistent with the results of our study, but there was no correlation analysis for vascular convergence. In addition, Zhou et al. (27) found that radiologic characteristics of enhanced CT images, including inverse variance, short axis, and extension, could be used as noninvasive predictors of the Ki-67 PI status in lung cancer patients. Therefore, according to the correlations between radiological features and Ki-67 PI, we could establish a radiological predictive model for $\mathrm{Ki}-67$, which is of great significance for prognostic stratification and clinical decision-making in LUAD. However, there are few related studies on the correlations between radiological features and Ki-67 PI at present. Therefore, further research is needed to provide a more sufficient reference for the establishment of the Ki-67 predictive model.

In terms of pathological features, we found that the patients with high $\mathrm{Ki}-67$ expression were mostly invasive LUAD $(\mathrm{P}<0.001)$, with a larger tumor tissue size $(\mathrm{P}<0.001)$, more VPI $(\mathrm{P}<0.001)$, LVI $(\mathrm{P}<0.001)$, lymph node metastasis $(\mathrm{P}<0.001)$, and a higher pathological TNM stage $(\mathrm{P}<0.001)$, which was consistent with previous reports $(10,28)$. Furthermore, we first analyzed the correlation between the 
STAS and Ki-67 PI and found that high expression of Ki67 was significantly related to the STAS $(\mathrm{P}=0.003)$. The WHO classification of lung cancer proposed the concept of STAS as a new pattern of invasion in LUAD in 2015 (17), and many studies have shown that the STAS is negatively correlated with the prognosis of LUAD $(29,30)$. These findings suggested that high Ki-67 expression could be associated with poor prognosis in patients with peripheral clinical stage IA LUAD.

In addition, there has been no definite research on the correlations between Ki-67 PI and the pathological subtypes of invasive LUAD. Only the study by Warth et al. (21) described that the average PI of Ki-67 was highest (39.4\%) in solid predominant LUAD, but there were no further statistical analyses. In the present study, we found that high Ki-67 expression was significantly associated with micropapillary predominant $(\mathrm{P}=0.040)$ and solid predominant LUAD $(\mathrm{P}<0.001)$. Low Ki-67 expression was more common in lepidic predominant LUAD $(\mathrm{P}<0.001)$. Previous studies have shown that lepidic predominant LUAD mostly manifests as GGO on CT imagines (31), while micropapillary and solid predominant LUAD is associated with solid nodules (32). Furthermore, we found that Ki-67 PI was significantly associated with CTR in the present study. Therefore, Ki-67 PI was consistent with the pathological subtype and radiological manifestation in patients with peripheral clinical stage IA LUAD.

However, when the pathological features were included in a multivariate analysis, we found that no relevant pathological feature was significantly associated with high expression of $\mathrm{Ki}-67$. As this study is a single-center retrospective study, further studies are needed to confirm the correlation between $\mathrm{Ki}-67$ and pathological features.

This study has several limitations. First, this study is a single-center retrospective study, and selection bias is inevitable. Second, Ki-67 immunohistochemical staining has not become a routine pathological detection procedure in LUAD patients, and many patients were excluded $(\mathrm{n}=238)$, which might have led to selection bias. Third, the cutoff value of Ki-67 PI based on patient prognosis was not determined. Therefore, we used the median value $(14 \%)$ as the cutoff value, similar to most previous studies. Fourth, the pathological data were based on review of postoperative reports, the parameters especially the Ki-67 PI could be influenced by subjectivity. While two separate pathologists were required to re-evaluated, the postoperative specimens could eliminate some of the subjectivity. Furthermore, assessing morphological features of pulmonary nodules is prone to inter and intra-observer variability. In addition, there was bias regarding the assessment knowing that these were proven tumors and the features mentioned are those that are relatively typical for malignant nodules.

\section{Conclusions}

Overall, we found that male sex, CEA positivity, notch positivity, vascular convergence, and CTR were significantly associated with high Ki-67 expression in peripheral clinical stage IA LUAD. These findings could be used to assist clinical decision-making and prognostic evaluation.

\section{Acknowledgments}

Funding: None.

\section{Footnote}

Reporting Checklist: The authors have completed the STROBE reporting checklist. Available at http://dx.doi. org/10.21037/tcr-20-2608

Data Sharing Statement: Available at http://dx.doi. org/10.21037/tcr-20-2608

Conflicts of Interest: All authors have completed the ICMJE uniform disclosure form (available at http://dx.doi. org/10.21037/tcr-20-2608). The authors have no conflicts of interest to declare.

Ethical Statement: The authors are accountable for all aspects of the work in ensuring that questions related to the accuracy or integrity of any part of the work are appropriately investigated and resolved. The study was conducted in accordance with the Declaration of Helsinki (as revised in 2013). The study was approved by the institutional ethics committee of China-Japan Friendship Hospital (No. 2018-13-K08). The institutional ethics committee waived the need for informed consent because of its retrospective design and the use of anonymized data.

Open Access Statement: This is an Open Access article distributed in accordance with the Creative Commons Attribution-NonCommercial-NoDerivs 4.0 International License (CC BY-NC-ND 4.0), which permits the noncommercial replication and distribution of the article with the strict proviso that no changes or edits are made and the 
original work is properly cited (including links to both the formal publication through the relevant DOI and the license). See: https://creativecommons.org/licenses/by-nc-nd/4.0/.

\section{References}

1. Gerdes J, Lemke H, Baisch H, et al. Cell cycle analysis of a cell proliferation-associated human nuclear antigen defined by the monoclonal antibody Ki-67. J Immunol 1984;133:1710-15.

2. Sobecki M, Mrouj K, Colinge J, et al. Cell-cycle regulation accounts for variability in $\mathrm{Ki}-67$ expression levels. Cancer Res 2017;77:2722-34.

3. Wu Q, Ma G, Deng Y, et al. Prognostic value of Ki-67 in patients with resected triple-negative breast cancer: a meta-analysis. Front Oncol 2019;9:1068.

4. Li H, Ren G, Cai R, et al. A correlation research of Ki67 index, CT features, and risk stratification in gastrointestinal stromal tumor. Cancer Med 2018;7:4467-74.

5. Yang F, Yu X, Bao Y, et al. Prognostic value of Ki-67 in solid pseudopapillary tumor of the pancreas: Huashan experience and systematic review of the literature. Surgery 2016;159:1023-31.

6. Kammerer-Jacquet SF, Ahmad A, Møller H, et al. Ki-67 is an independent predictor of prostate cancer death in routine needle biopsy samples: proving utility for routine assessments. Mod Pathol 2019;32:1303-9.

7. Ziaran S, Harsanyi S, Bevizova K, et al. Expression of E-cadherin, Ki-67, and p53 in urinary bladder cancer in relation to progression, survival, and recurrence. Eur J Histochem 2020;64:3098.

8. Bray F, Ferlay J, Soerjomataram I, et al. Global cancer statistics 2018: GLOBOCAN estimates of incidence and mortality worldwide for 36 cancers in 185 countries. CA Cancer J Clin 2018;68:394-424.

9. Lee YS, Bae SC. How do K-RAS-activated cells evade cellular defense mechanisms? Oncogene 2016;35:827-32.

10. Wei DM, Chen WJ, Meng RM, et al. Augmented expression of Ki-67 is correlated with clinicopathological characteristics and prognosis for lung cancer patients: an up-dated systematic review and meta-analysis with 108 studies and 14,732 patients. Respir Res 2018;19:150.

11. Li X, Liu J, Qian L, et al. Expression of PFKFB3 and $\mathrm{Ki} 67$ in lung adenocarcinomas and targeting PFKFB3 as a therapeutic strategy. Mol Cell Biochem 2018;445:123-34.

12. Wen $\mathrm{S}, \mathrm{Zhou} \mathrm{W,} \mathrm{Li} \mathrm{CM}$, et al. Ki-67 as a prognostic marker in early-stage non-small cell lung cancer in Asian patients: a meta-analysis of published studies involving 32 studies. BMC Cancer 2015;15:520.

13. Xu J, Liu P, Da J, et al. Prognostic value of Ki-67 in stage I non-small-cell lung cancer: a meta-analysis involving 1931 patients. Pathol Res Pract 2019;215:855-60.

14. Yamashita S, Moroga T, Tokuishi K, et al. Ki-67 labeling index is associated with recurrence after segmentectomy under video-assisted thoracoscopic surgery in stage I non-small cell lung cancer. Ann Thorac Cardiovasc Surg 2011;17:341-6.

15. Goldstraw P, Chansky K, Crowley J, et al. The IASLC Lung Cancer Staging Project: proposals for revision of the TNM stage groupings in the forthcoming (eighth) edition of the TNM classification for lung cancer. J Thorac Oncol 2016;11:39-51.

16. Travis WD, Brambilla $\mathrm{E}$, Noguchi $M$, et al. International Association for the Study of Lung Cancer/American Thoracic Society/European Respiratory Society: international multidisciplinary classification of lung adenocarcinoma: executive summary. Proc Am Thorac Soc 2011;8:381-5.

17. Travis WD, Brambilla E, Nicholson AG, et al. The 2015 World Health Organization Classification of lung tumors: impact of genetic, clinical and radiologic advances since the 2004 classification. J Thorac Oncol 2015;10:1243-60.

18. Collins J, Stern EJ. Ground-glass opacity at CT: the ABCs. AJR Am J Roentgenol 1997;169:355-67.

19. Goldhirsch A, Wood WC, Coates AS, et al. Strategies for subtypes--dealing with the diversity of breast cancer: highlights of the St. Gallen International Expert Consensus on the Primary Therapy of Early Breast Cancer 2011. Ann Oncol 2011;22:1736-47.

20. Rindi G, Falconi M, Klersy C, et al. TNM staging of neoplasms of the endocrine pancreas: results from a large international cohort study. J Natl Cancer Inst 2012;104:764-77.

21. Warth A, Cortis J, Soltermann A, et al. Tumour cell proliferation (Ki-67) in non-small cell lung cancer: a critical reappraisal of its prognostic role. Br J Cancer 2014;111:1222-9.

22. Kim CH, Lee HS, Park JH, et al. Prognostic role of p53 and Ki-67 immunohistochemical expression in patients with surgically resected lung adenocarcinoma: a retrospective study. J Thorac Dis 2015;7:822-33.

23. Oka S, Uramoto H, Shimokawa H, et al. The expression of $\mathrm{Ki}-67$, but not proliferating cell nuclear antigen, predicts poor disease free survival in patients with adenocarcinoma of the lung. Anticancer Res 2011;31:4277-82. 
24. Mikkonen L, Pihlajamaa P, Sahu B, et al. Androgen receptor and androgen-dependent gene expression in lung. Mol Cell Endocrinol 2010;317:14-24.

25. Stakišaitis D, Mozūraite R, Kavaliauskaitè D, et al. Sexrelated differences of urethane and sodium valproate effects on Ki-67 expression in urethane-induced lung tumors of mice. Exp Ther Med 2017;13:2741-50.

26. Yan J, Wang H, Zhou H, et al. Correlation between expression of Ki-67 and MSCT signs in different types of lung adenocarcinoma. Medicine (Baltimore) 2020;99:e18678.

27. Zhou B, Xu J, Tian Y, et al. Correlation between radiomic features based on contrast-enhanced computed tomography images and Ki-67 proliferation index in lung cancer: a preliminary study. Thorac Cancer 2018;9:1235-40.

28. He LY, Zhang H, Wang ZK, et al. Diagnostic and prognostic significance of E-cadherin and Ki-67 expression

Cite this article as: Liu Z, Feng $\mathrm{H}$, Ma S, Shao W, Zhang J, Zhang Z, Sun H, Gu X, Zhang Z, Liu D. Clinicopathological characteristics of peripheral clinical stage IA lung adenocarcinoma with high Ki-67 expression. Transl Cancer Res 2021;10(1):152-161. doi: 10.21037/tcr-20-2608 in non-small cell lung cancer patients. Eur Rev Med Pharmacol Sci 2016;20:3812-7.

29. Uruga H, Fujii T, Fujimori S, et al. Semiquantitative assessment of tumor spread through air spaces (STAS) in early-stage lung adenocarcinomas. J Thorac Oncol 2017:12:1046-51.

30. Toyokawa G, Yamada Y, Tagawa T, et al. Significance of spread through air spaces in resected pathological stage I lung adenocarcinoma. Ann Thorac Surg 2018:105:1655-63.

31. Pedersen JH, Saghir Z, Wille MM, et al. Groundglass opacity lung nodules in the era of lung cancer CT screening: radiology, pathology, and clinical management. Oncology (Williston Park) 2016;30:266-74.

32. Cha MJ, Lee HY, Lee KS, et al. Micropapillary and solid subtypes of invasive lung adenocarcinoma: clinical predictors of histopathology and outcome. J Thorac Cardiovasc Surg 2014;147:921-8.e2. 